\title{
Sets in the Plane with Many Concyclic Subsets
}

\author{
R.H. Jeurissen \\ Mathematical Institute, Radboud University \\ Toernooiveld, Nijmegen, The Netherlands \\ R. Jeurissen@science.ru.nl \\ Submitted: Jul 23, 2003; Accepted: Aug 7, 2005; Published: Aug 30, 2005 \\ Mathematics Subject Classification: 05B30, 51M04
}

\begin{abstract}
We study sets of points in the Euclidean plane having property $R(t, s)$ : every $t$-tuple of its points contains a concyclic $s$-tuple. Typical examples of the kind of theorems we prove are: a set with $R(19,10)$ must have all its points on two circles or all its points, with the exception of at most 9 , are on one circle; of a set with $R(8,5)$ and $N \geq 28$ points at least $N-3$ points lie on one circle; a set of at least 109 points with $R(7,4)$ has $R(109,7)$. We added some results on the analogous configurations in 3-space.
\end{abstract}

\section{Introduction}

If all points, or all points but one, of a set $V$ of points in the Euclidean plane are on a circle, then clearly every 5 -subset of $V$ contains a concyclic 4-subset. In [2] it was proved that the converse also holds, unless $|V|=6$. In [1] other proofs were given and also the following was proved. If every 6-subset of a set $V,|V|>77$, of points in the Euclidean plane contains a concyclic 4-subset, then all points of $V$ with the exception of at most two are on a circle. The same then must hold if the condition is strengthened to: every 7 -subset contains a concyclic 5-subset. We shall see below (Proposition 6) that then the condition $|V|>77$ can be omitted. More generally we investigate sets satisfying the condition one gets by replacing the pair $(7,5)$ by $(t, s), t \geq s>3$.

It may be noteworthy that the essential point of the proofs in [1] and [2] is that the $2-(7,4,2)$ design (the complementary design of the $2-(7,3,1)$ design) has no realisation in the plane with concyclic quadruples as blocks. This means that there is no configuration

of 7 points and 7 circles such that every circle contains 4 of the points and every pair of circles intersect in 2 of the points. 


\section{Preliminaries and Examples}

Above, where we wrote "concyclic" and "circle" one may read "concyclic or collinear" and "circle or line", respectively. The reason is that the only property of the set of circles that plays a role is that its elements are determined by three of their points (in [1], but not below, it is also used that two pairs of points on a circle do or do not separate each other). The same holds for the set of circles and lines and for any subset of that set. So in what follows (except in Section 7), to avoid lengthy expressions, we shall silently assume that there is a prescribed set $\mathcal{S}$ of circles and/or lines, and call a set round if it has all its points on a circle or line of $\mathcal{S}$. Its support will be that line or that circle. (The reader may still prefer to think of $\mathcal{S}$ as the set of all circles and accordingly read "round" as "concyclic".)

By set we will always mean a set of points in the Euclidean plane (except in Section 8). $V$ will denote such a set and $N$ its cardinality. We say that $V$ has property $R(t, s)$ if $3<s \leq t \leq N$ and if each of its $t$-subsets contains a round $s$-subset. (Sets with $R(s, s)$, $s>3$, are easily seen to be round and of course $N=t$ is also a trivial case, but admitting these cases allows for easier formulations.)

A set has property $C(r)$ if there is a round set containing all its points with the exception of at most $r$.

Trivial examples of sets with $R(t, s)$ are sets with $C(t-s)$. So we can state the theorems mentioned in the introduction in a condensed form as follows. If $R(5,4)$ and $N>6$ then $C(1)$ and if $R(6,4)$ and $N>77$ then $C(2)$. (The validity for infinite $N$ easily follows from that for finite $N$. This being also the case for all theorems below the reader may find it comfortable to think of finite sets only.)

Lemma 1 A set has property $R(t, s)$ if it is the union of $k$ round sets, $k>0$, and a set of $p$ points, where $p<t-k(s-1)$.

Proof. A $t$-subset has at least $t-p$ of its points in the union of the $k$ round sets. Since $t-p>k(s-1)$, at least one of the round sets must contain at least $s$ of these $t-p$ points. (Note that $k=0$ would be the excluded case $N<t$ and that the round sets or their supports may have points in common.)

$C(t-s)$ is the case $k=1$ in the lemma. Another special case is that with $p=0$ : all points are on $k$ round sets and $k(s-1)<t$.

In Figure 1 we see examples (with $\mathcal{S}$ the set of all circles and lines) of a 12 -set with $R(6,4)$, a 10 -set with $R(8,5)$, a 9 -set with $R(8,5)$ and an 11-set with $R(6,4)$, all escaping the condition in the lemma.

A set satisfying the condition in the lemma, thus with $R(t, s)$ in a rather trivial way, will be said to have property $R^{*}(t, s)$. If $2 s \geq t+2$ we can only have $R^{*}(t, s)$ with $k=1$, thus if we have $C(t-s)$.

Clearly $R(t, s)$ entails $R(t+1, s)$ and, if $s>4, R(t, s-1)$ and $R(t-1, s-1)$. The same holds with $R^{*}$ instead of $R$.

The first example above has $R(6,4)$ so it has $R(7,4)$. It has not $R^{*}(6,4)$ but it has $R^{*}(7,4)$. The third example has $R(8,5)$ but not $R^{*}(8,5)$; it has $R^{*}(8,4)$, however. 


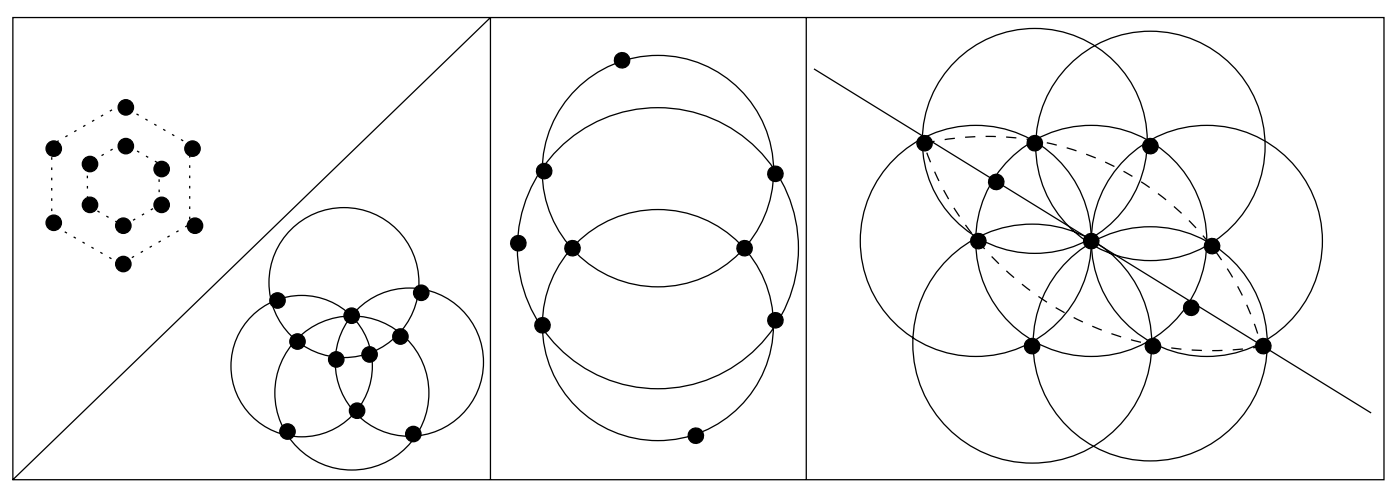

Figure 1: Examples

It is easy to construct examples for large $t$ :

Lemma 2 If $V_{1}$ and $V_{2}$ are disjoint sets with $R\left(t_{1}, s\right)$ and $R\left(t_{2}, s\right)$, respectively, then $V_{1} \cup V_{2}$ has $R\left(t_{1}+t_{2}-1, s\right)$. The same holds with $R^{*}$ instead of $R$.

Proof. The $R$-case: a $\left(t_{1}+t_{2}-1\right)$-subset of $V_{1} \cup V_{2}$ contains a $t_{1}$-subset of $V_{1}$ or it contains a $t_{2}$-subset of $V_{2}$, so it contains a round $s$-subset of $V_{1}$ or a round $s$-subset of $V_{2}$. The $R^{*}$ case: if $V_{i}, i=1,2$, consists of $k_{i}$ round sets and a $p_{i}$-set with $p_{i}<t_{i}-k_{i}(s-1)$, then $V_{1} \cup V_{2}$ consists of $k_{1}+k_{2}$ round sets and a $\left(p_{1}+p_{2}\right)$-set with $p_{1}+p_{2}<t_{1}+t_{2}-1-\left(k_{1}+k_{2}\right)(s-1)$.

Corollary 3 If $V$ is a set with $R\left(t_{1}, s_{1}\right)$ and $W$ is a set with $R\left(t_{2}, s_{2}\right)$, then $V \cup W$ has $R\left(t_{1}+t_{2}-1, \min \left(s_{1}, s_{2}\right)\right)$. The same holds with $R^{*}$ instead of $R$.

In the $R^{*}$ cases a certain reverse also holds:

Lemma 4 If $Q$ is a set with $R^{*}(t, s)$, then i) $Q$ has $C(t-s)$ or ii) $Q$ is the union of at least 2 and at most $\left\lfloor\frac{t-1}{s-1}\right\rfloor$ round sets or iii) $Q$ is the union of a set $V$ with $R^{*}(s+1, s)$ and a set $W$ with $R^{*}(t-s, s)$.

Proof. $Q$ consists of $k$ round sets and a set $P$ with $p$ points, where $p<t-k(s-1)$ (and $k>0$ ). We may suppose the round sets to be disjoint, assigning common points to only one of them. If one of the round sets has less than $s$ points (and thus $k>1$ ) we add its points to $P$ to get $k-1$ round sets and a set with at most $p+s-1<t-(k-1)(s-1)$ points. So we may suppose the round sets to have cardinality $\geq s$. If now $k=1$ we have $C(t-s)$. If $k>1$ and $p=0$ we have $k(s-1)<t$, and hence $k \leq\left\lfloor\frac{t-1}{s-1}\right\rfloor$. If $k>1$ and $p>0$ let $V$ consist of one of the round sets and one point $p$ from $P$. Then $V$ has $C(1)$, so $R^{*}(s+1, s)$. $W:=Q-V$ consists of $k-1(\geq 1)$ round sets and a set with $p-1$ points. Now $p-1<t-s-(k-1)(s-1)$ so if $|W| \geq t-s$ then $W$ has $R^{*}(t-s, s)$; else $|W \cup\{p\}| \leq t-s$ so $Q$ has $C(t-s)$. 
The three possibilities in the lemma need not exclude each other. If $Q$ is the union of two round sets of 7 points that have 2 points in common $(\mathrm{N}=12)$ it has $R^{*}(10,5)$ and $\left.\mathrm{i}\right)$, ii) and iii) are all true. However for the union of four round 6-sets with disjoint supports, which has $R^{*}(21,6)$, only ii) is true.

In the $R$-case also a reverse holds, provided the set is sufficiently large. We return to this in section 6 .

\section{Simple Cases}

Proposition 5 Let $2 s \geq t+4$. Then every set that has property $R(t, s)$ has property $C(t-s)$.

Proof. A set $V$ with $R(t, s)$ has a round $s$-subset $W$, say $W=\{1,2, \ldots, s\}$. If $C(t-s)$ does not hold, $V$ has a $(t-s+1)$-subset $U=\{s+1, s+2, \ldots, t+1\}$ of points not on the support of $W$. The $t$-subset $T=(W-\{1\}) \cup U$ contains a round $s$-subset that can not contain 3 points of $W$, so it contains $\geq s-2$ points of $U$. Then $s-2 \leq|U|=t-s+1$, so $2 s \leq t+3$, a contradiction.

Proposition 6 Let $2 s=t+3$ and $s \geq 5$. Then every set with property $R(t, s)$ has property $C(t-s)$.

Proof. Suppose $C(t-s)=C(s-3)$ does not hold and take $W, U$ and $T$ as in the previous proof; $|U|=s-2 \geq 3$. $T$ contains a round $s$-set $S$ containing $U$ and exactly 2 points of $W$, say $S=\{2,3\} \cup U$. Likewise $T^{\prime}=(W-\{2\}) \cup U$ contains a round $s$-set $S^{\prime}=\{v, w\} \cup U,\{v, w\} \neq\{2,3\}$. Hence the support of $U$ contains $\geq 3$ points of $W$, so that it coincides with the support of $W$, contradiction.

This deals with the case $R(7,5)$, mentioned in the Introduction. As is mentioned there the condition $s \geq 5$ is not needed if the set has more than 6 points, but without it the set of the six intersection points of three circles, which has $R(5,4)$, would be a counterexample (in fact the only one).

Proposition 7 Let $2 s=t+2$ and $s \geq 6$. Then every set that has property $R(t, s)$ has property $C(t-s)$.

Proof. Suppose that $C(t-s)=C(s-2)$ does not hold and take $W, U$ and $T$ as in the previous proofs; $|U|=s-1 \geq 5$.

If $U$ is not round we may suppose the round $s$-subset in $T$ to be $\{2,3\} \cup(U-\{t+1\})$. In $T^{\prime}=(W-\{2\}) \cup U$ there is a round $s$-set of the form $\{x, y\} \cup(U-\{z\})$ with $\{x, y\} \neq\{2,3\}$. Since $U-\{t+1\}$ and $U-\{z\}$ are round but $U$ is not, we have $z=t+1$. The support of $U-\{t+1\}$ thus contains $\geq 3$ points of that of $W$. Contradiction as in the previous proof.

If $U$ is round, then we see from $T$ that at least one point of $W$ is on the support of $U$, say 2. With $T^{\prime}$ it then follows that there is a second point, say 1. If a point $q \in V-(W \cup U)$ 
would be on the support of $W$, then $R(t, s)$ would not hold, see $\{q, 3,4, \ldots, s\} \cup U$. If all points of $V-(W \cup U)$ would be on the support of $U$, then $C(s-2)$ would hold. So there is a point $q \in V$ not on the support of $W$ or on the support of $U$. But then we can, instead of $U$, take the not round $(U-\{t+1\}) \cup\{q\}$ and finish the proof as before.

The third example above has $R(8,5)$ and shows that the condition $s \geq 6$ can not be omitted.

Note that the last three propositions cover all cases with $2 s \geq t+2$ (i.e. the cases where the properties $R^{*}(t, s)$ and $C(t-s)$ coincide) except for $(t, s)=(5,4),(t, s)=(6,4)$ and $(t, s)=(8,5)$. For the first two cases see the Introduction. The third case will be dealt with in Section 5 .

If $2 s \leq t+1$ and $t$ is sufficiently small we also have only simple cases:

Proposition 8 Let $2 s-1 \leq t \leq 3 s-8$ (and consequently $s \geq 7$ ). Then every set with $R(t, s)$ has $R^{*}(t, s)$.

Proof. Let $V$ be such a set. Take a round $s$-set $W=\{1,2, \ldots, s\}$ in $V$ and let $C$ be its support. Let $Q=V-(V \cap C)$. If $|Q| \leq t-s$ we have $C(t-s)$, thus $R^{*}(t, s)$. Next let $|Q| \geq t-s+1$.

1) We first prove that $Q$ has property $R(t-s+1, s-1)$. Let $U=\{s+1, s+2, \ldots, t+1\}$ be an arbitrary $(t-s+1)$-subset of $Q$. Suppose $U$ contains no round $(s-1)$-set. Let $T=(W \cup U)-\{s\}$ so $|T|=t$. $T$ contains a round $s$-set $S$ for which we must have $|S \cap W| \leq 2$ and $|S \cap U| \leq s-2$, so we can suppose that $S=\{1,2\} \cup R, R$ an $(s-2)$ subset of $U$. Now let $T^{\prime}=(W \cup U)-\{1\}$. For a round $s$-set $S^{\prime}$ in $T^{\prime}$ we likewise have $S^{\prime}=\{x, y\} \cup R^{\prime},\{x, y\} \subseteq W,\{x, y\} \neq\{1,2\}$ and $R^{\prime}$ an $(s-2)$-subset of $U$. Now $\left|R \cap R^{\prime}\right| \leq 2$, otherwise $S$ and $S^{\prime}$ would have the same support, which would share $\{1,2, x, y\}$ with $\mathrm{C}$, so be $C$, whereas $R \cap C=\emptyset$. So $R^{\prime}$ must contain at least $s-4$ points of $U-R$. Since $|U-R|=t-2 s+3 \leq s-5$, this is impossible.

2) Now $2(s-1) \geq(t-s+1)+5$ so $Q$ has $C(t-2 s+2)$ by Proposition 5 . So $V$ is the union of a round set $R_{1}$ with support $C_{1}$ and with $\left|R_{1}\right| \geq s$, a second round set $R_{2}$ disjoint from $C_{1}$ with support $C_{2}$ and with $\left|R_{2}\right| \geq s-1$ and a set $R_{3}$ of at most $t-2 s+2$ points disjoint from $C_{1} \cup C_{2}$. If $\left|R_{3}\right|<t-2(s-1)$ we have $R^{*}(t, s)$, so now we assume that $\left|R_{3}\right|=t-2 s+2$.

3) Let $X_{i}$ be an $(s-1)$-set in $R_{i}, i=1,2$. Let $X$ be the $t$-set $X_{1} \cup X_{2} \cup R_{3}$. A round $s$-set in $X$ can contain at most 2 points from $X_{1}$ so it contains at least $s-2-\left|R_{3}\right|=3 s-t-4>3$ points of $X_{2}$. So its support is $C_{2}$ and it thus contains at least one point of $C_{2} \cap X_{1}$. Let $x$ be such a point. Likewise, after replacing $x$ in $X_{1}$ by a point of $R_{1}-X_{1}$, we find a second point $y$ in $C_{2} \cap X_{1}$. Replacing $R_{1}$ by $R_{2} \cup\{x, y\}$ and $R_{2}$ by $R_{1} \backslash\{x, y\}$ we may now assume that $\left|R_{1}\right| \geq s+1$ and $\left|R_{2}\right| \geq s-2$.

4) If $\left|R_{2}\right|>s-2$ we can make a $t$-set by taking $s-1$ points from each of $R_{1}$ and $R_{2}$ and all points from $R_{3}$ without using the (at most 2) points of $R_{1} \cap C_{2}$. This $t$-set does not contain a cyclic $s$-set, since $2+2+t-2 s+2=t-2 s+6<s$. So $\left|R_{2}\right|=s-2$ and we have $C(t-s)$ since $s-2+t-2 s+2=t-s$. 
Remark Following a suggestion of a referee we could replace "round" by "collinear" in the definition of $R(t, s)$. Only small changes in the proof of Proposition 5 suffice to show the following. Every set with the property that each $t$-subset contains a collinear $s$-set has all its points except for at most $t-s$ on a line, provided that $2 s \geq t+3$. The same holds if $2 s=t+2$ (use the proof of Proposition 6). Two parallel collinear sets of $s$ points each are a counterexample for the case $2 s=t+1$.

\section{Large Round Sets}

The theorems mentioned in the Introduction could suggest that a set with $R(t, s)$, if sufficiently large, is trivial in the sense that it has $R^{*}(t, s)$. This is of course true for the pairs $(t, s)$ treated in Section 3 but there are still other such pairs as we shall show in Section 5. In Section 6 we derive some consequences of $R(t, s)$ in general for sufficiently large sets.

That at least large round subsets can not be avoided when we increase $|V|$ can be shown using the Ramsey numbers $\operatorname{Ram}(p, q ; 4)$. Indeed, if $|V| \geq \operatorname{Ram}(n, t-s+4 ; 4)$ for a set $V$ with $R(t, s)$, then $V$ has an $n$-subset in which all 4-tuples are round, so that it is itself round, or a $(t-s+4)$-subset $U$ in which no 4 -tuple is round. The latter is impossible: adding $s-4$ points to $U$ would give a $t$-tuple which would contain a round $s$-tuple with at least 4 points in $U$. We want a more concrete bound, however.

Theorem 9 Let $V$ be a set with property $R(t, s)$, let $N=|V|$ and let $d$ and $q$ be integers with $3 \leq d<q \leq s$. Then $V$ contains a round $n$-set if

$$
\left(\begin{array}{l}
N-d \\
q-d
\end{array}\right)\left(\begin{array}{l}
s \\
q
\end{array}\right)>\left(\left(\begin{array}{l}
n-d \\
q-d
\end{array}\right)-1\right)\left(\begin{array}{l}
t \\
q
\end{array}\right) .
$$

Proof. We shall prove that there is a $d$-subset of $V$ that is contained in $\left(\begin{array}{l}n-d \\ q-d\end{array}\right)$ round $q$-subsets of $V$; their union then is a round set of cardinality $\geq n$. Let $r$ be the number of round $q$-subsets in $V$. Suppose every $d$-subset of $V$ is contained in at most $m=\left(\begin{array}{l}n-d \\ q-d\end{array}\right)-1$ of these subsets. Counting in two ways pairs $\langle Q, T\rangle$ with $Q$ a round $q$-set, $T$ a $t$-set and $Q \subset T \subseteq V$, we find, since every $t$-subset contains a round $s$-set,

$$
r\left(\begin{array}{c}
N-q \\
t-q
\end{array}\right) \geq\left(\begin{array}{c}
N \\
t
\end{array}\right)\left(\begin{array}{l}
s \\
q
\end{array}\right) .
$$

Counting in two ways pairs $\langle D, Q\rangle$ with $D$ a $d$-set, $Q$ a round $q$-set and $D \subset Q \subseteq V$, we find

$$
r\left(\begin{array}{l}
q \\
d
\end{array}\right) \leq m\left(\begin{array}{l}
N \\
d
\end{array}\right)
$$

From (1) and (2):

$$
\left(\begin{array}{l}
N \\
t
\end{array}\right)\left(\begin{array}{l}
s \\
q
\end{array}\right)\left(\begin{array}{l}
q \\
d
\end{array}\right) \leq m\left(\begin{array}{l}
N \\
d
\end{array}\right)\left(\begin{array}{c}
N-q \\
t-q
\end{array}\right)
$$


from which:

$$
\left(\begin{array}{c}
N-d \\
q-d
\end{array}\right)\left(\begin{array}{l}
s \\
q
\end{array}\right) \leq m\left(\begin{array}{l}
t \\
q
\end{array}\right)
$$

contradicting the condition in the theorem.

For many triples $\langle t, s, n\rangle$ the lowest bound for $N$ will appear if we take $d=3$ and $q=s$; if $\mathrm{s}=4$ this is the only choice. So we state:

Corollary 10 An $N$-set with $R(t, s)$ contains a round n-set if

$$
\left(\begin{array}{c}
N-3 \\
s-3
\end{array}\right)>\left(\left(\begin{array}{c}
n-3 \\
s-3
\end{array}\right)-1\right)\left(\begin{array}{l}
t \\
s
\end{array}\right)
$$

In particular a set with $R(t, 4)$ and with $N$ points contains a round $n$-set if

$$
N>3+(n-4)\left(\begin{array}{l}
t \\
4
\end{array}\right) .
$$

However to guarantee a round 100-set in a set $V$ with $R(14,7)$, for instance, we need $|V| \geq 736$ according to the corollary but the theorem with $d=3$ and $q=6$ gives the better bound $|V| \geq 729$. For a 500-set we find 3798 and 3745, respectively. (Calculations carried out by MAPLE.)

To get a simpler formula we could take $d=q-1$ in the theorem:

$$
N>q-1+(n-q)\left(\begin{array}{l}
t \\
q
\end{array}\right)\left(\begin{array}{l}
s \\
q
\end{array}\right)^{-1} .
$$

The right hand side increases with $q$ so we better take $q=4$ :

$$
N>3+(n-4)\left(\begin{array}{l}
t \\
4
\end{array}\right)\left(\begin{array}{l}
s \\
4
\end{array}\right)^{-1}
$$

By using that $R(t, s)$ entails $R\left(t-s+4,4\right.$ ) (or that $\left(\begin{array}{c}t-s+4 \\ 4\end{array}\right) \geq\left(\begin{array}{l}t \\ 4\end{array}\right)\left(\begin{array}{l}s \\ 4\end{array}\right)^{-1}$ if $s \geq 4$, by induction on $t$ ) we get:

$$
N>3+(n-4)\left(\begin{array}{c}
t-s+4 \\
4
\end{array}\right) .
$$

Probably our condition is far too strong. Indeed, whereas, as mentioned in the Introduction, a set $V$ with $R(5,4)$ contains a round $n$-set if $|V|>n>5$, the theorem only promises a round $n$-set if $|V|>5 n-17$. By the theorem (take $d=3$ and $q=6$ ) a 29-set with $R(10,7)$ has a round 12 -set, but by Proposition 5 this is already true for a 15-set.

The proof in [1] that a set $V$ with $R(6,4)$ and $|V| \geq 78$ has $C(2)$ is based on a lemma ([1], Lemma 2) stating that such a set contains a round 9-set. The corollary guarantees a round 9-set if $|V|>78$. The next theorem however will tell us that $|V| \geq 78$ is sufficient. Contrary to Theorem 9 it gives a direct (lower) bound for $n$ when $N$ is given. 
Theorem 11 Let $V$ be a set with property $R(t, s)$ and let $|V| \geq N$. Let $3<q \leq s$. Then $V$ contains a round subset of cardinality

$$
\left.\left\lceil\cdots\left\lceil\left\lceil\left\lceil\left(\begin{array}{l}
N \\
q
\end{array}\right)\left(\begin{array}{l}
s \\
q
\end{array}\right)\left(\begin{array}{l}
t \\
q
\end{array}\right)^{-1}\right\rceil \frac{q}{N}\right\rceil \frac{q-1}{N-1}\right\rceil \frac{q-2}{N-2}\right\rceil \cdots \frac{2}{N-q+2}\right\rceil+q-1 .
$$

Proof. Let $V^{\prime}$ be a subset of $V$ of cardinality $N$. Then $V^{\prime}$ also has $R(t, s)$. The number of round $q$-subsets in $V^{\prime}$ is (see (1) in the proof of Theorem 9) $\geq\left(\begin{array}{c}N \\ t\end{array}\right)\left(\begin{array}{c}s \\ q\end{array}\right)\left(\begin{array}{c}N-q \\ t-q\end{array}\right)^{-1}=$ $\left(\begin{array}{l}N \\ q\end{array}\right)\left(\begin{array}{l}s \\ q\end{array}\right)\left(\begin{array}{l}t \\ q\end{array}\right)^{-1}$, so it is at least $T_{q}:=\left\lceil\left(\begin{array}{l}N \\ q\end{array}\right)\left(\begin{array}{l}s \\ q\end{array}\right)\left(\begin{array}{l}t \\ q\end{array}\right)^{-1}\right\rceil$. So there is a $T_{q} \times N 0,1$-matrix $M_{0}$ of which the rows are the characteristic vectors of different round $q$-subsets. Since that matrix contains $T_{q} \cdot q 1$ 's, there is a column, say the first, with $\geq T_{q-1}:=\left\lceil T_{q} \frac{q}{N}\right\rceil 1$ 's. Deleting the rows with a 0 in the first column and then also the first column we get a submatrix $M_{1}$ with $\geq T_{q-1}(q-1)$ 1's, so there is a column, say again the first, with $\geq T_{q-2}:=\left\lceil T_{q-1} \frac{q-1}{N-1}\right\rceil=\left\lceil\left\lceil T_{q} \frac{q}{N}\right\rceil \frac{q-1}{N-1}\right\rceil 1$ 's. Continuing in the same way we finally find a submatrix $M_{q-1}$ with $T_{1}:=\left\lceil\cdots \cdots\left\lceil\left\lceil\left\lceil\left\lceil\left(\begin{array}{c}N \\ q\end{array}\right)\left(\begin{array}{c}s \\ q\end{array}\right)\left(\begin{array}{c}t \\ q\end{array}\right)^{-1}\right\rceil \frac{q}{N}\right\rceil \frac{q-1}{N-1}\right\rceil \frac{q-2}{N-2}\right\rceil \cdots \cdots \frac{2}{N-q+2}\right\rceil$ rows each containing a 1 in a different column. They correspond to $T_{1}$ round $q$-subsets sharing $q-1(\geq 3)$ points. The union of these sets is a round $T_{1}+q-1$ set.

The best choice for $q$ seems to be 4 . The theorem guarantees a round 20-set in a set with $R(20,10)$ having 374 points, in a set with $R(13,6)$ having 766 points and in a set with $R(13,7)$ having 330 points. These results are poor compared with the cardinalities one gets by Theorem 9: 79, 196 and 104, respectively. Moreover in the third case a cardinality 40 is already sufficient, as follows from Proposition 8.

A less precise proof we get by omitting the inner ceilings. It yields the cardinality

$\left\lceil(N-q+1)\left(\begin{array}{l}s \\ q\end{array}\right)\left(\begin{array}{l}t \\ q\end{array}\right)^{-1}\right\rceil+q-1$, so a round $n$-set if $N>(n-q)\left(\begin{array}{l}t \\ q\end{array}\right)\left(\begin{array}{l}s \\ q\end{array}\right)^{-1}+q-1$. This is precisely the bound in (3) we got by taking $d=q-1$ in Theorem 9 , what suggests that generally as with that bound the best choice is $q=4$, but also that Theorem 9 will give a better result if $s>4$. But if $s=4$ using the ceil's we may gain a little. For instance with $R(6,4)$ a round set of 9 points is guaranteed by Theorem 9 if $N \geq 79$ and by Theorem 11 if $N \geq 78$. A round 5-set then exists according to Theorem 9 if $N \geq 19$, but according to Theorem 11 if $N \geq 17$.

\section{Some Particular Cases}

$R(7,4)$ is the "smallest" case in which $k$ (as in Lemma 1) can be 2 . The second example in Figure 1 has $R(7,4)$ with $N=10$, but not $R^{*}(7,4)$.

Proposition 12 A set with $R(7,4)$ having a round 27-subset or a cardinality $\geq 809$ has $R^{*}(7,4)$ (i.e. consists of one or two round sets).

Proof. Such a set $V$ contains a round subset with 27 points, by Corollary 10 as well as by Theorem 11. Let $C$ be its support. Let $P=V \cap C$, so $|P| \geq 27$, and $Q=V-P$. If $Q$ is a 
round or empty set we are ready. If not, then $Q$ has a non-round 4-subset $T=\{a, b, c, d\}$. A round set through 3 points of $T$ contains at most 2 points of $P$, so $P$ has a subset $U$ of (at least) 19 points none of which form a round set with 3 points from $T$. This gives us $\left(\begin{array}{c}19 \\ 3\end{array}\right)=9697$-tuples $\{i, j, k, a, b, c, d\}$ with $\{i, j, k\} \subset U$, each containing a round 4-tuple. Such a 4-tuple must have 2 points in $U$ and 2 points in $T$. Since in $T$ there are only 6 pairs, there is a pair, $\{a, b\}$ say, that is part of a round 4-tuple in $\geq 969 / 6$, so in at least 162, of our 7-tuples. But for instance $\{1,2, a, b\}$ and $\{1,3, a, b\}$ can not both be round, since then $a$ and $b$ would be on $C$. So there are at most 9 round 4 -tuples $\{i, j, a, b\}$ with $i, j \in U$, and one of them must occur in $\geq 162 / 9=18$ of our 7 -tuples. Since, however, for given $i$ and $j$ there are only 17 triples $\{i, j, k\}$ in $U$ this is impossible.

With the stronger property $R(8,5)$ a smaller cardinality is sufficient:

Proposition 13 A set with $R(8,5)$ having a round 7 -subset or a cardinality $\geq 28$ has $C(3)$.

Proof. By Corollary 10 such a set $V$ has a round subset $P$ with 7 points, $1,2 \ldots, 7$, say. Let $C$ be its support and $Q$ the set of points of $V$ not on $C$. Suppose $|Q|>3$ and let $U=\{a, b, c, d\}$ be a 4-subset of $Q$. If $U$ is round its support has at most 2 points in $P$, say 7 , or 6 and 7 , if any. But then there is no round 5 -set in $\{1,2,3,4, a, b, c, d\}$, so $U$ is not round. The triples from $U$ determine 4 circles (or 3 circles and one line) of which no two can pass through the same point of $P$ (if, e.g., $\{a, b, c, 1\}$ and $\{a, b, d, 1\}$ would be round, then $U$ would be round). So at most 3 of these round sets contain a pair of points of $P$. Deleting from $P$ one point of every such pair we see that there is a 4 -tuple in $P$ having no two of its points on one of these four round sets; let $\{1,2,3,4\}$ be such a 4-tuple. Then $\{1,2,3,4, a, b, c, d\}$ would not contain a round 5-tuple. So $|Q| \leq 3$ and we have $C(3)$.

The proof only uses $R(8,5)$ and the existence of a round 7-set, so could be also used in the $R(9,6)$-case $(R(9,6)$ entails $R(8,5)$ and guarantees a round 7 -set already if there are 16 points). But the case $R(9,6)$ is better treated by Proposition 6 .

Proposition $14 A$ set with $R(9,5)$ having a round 12-subset or a cardinality $\geq 98$ has $R^{*}(9,5)$.

Proof. Such a set $V$ has a round subset $P$ with 12 points by Corollary 10 . Let $C$ be its support and $Q$ the set of points of $V$ not on $C$. We are ready if $|Q|<5$, so we suppose $|Q| \geq 5$.

First assume $Q$ has a 5 -subset $T=\{a, b, c, d, e\}$ not containing a round 4-tuple. There are $\left(\begin{array}{c}12 \\ 4\end{array}\right)=495$ 9-tuples $\{h, i, j, k, a, b, c, d, e\}$ with $\{h, i, j, k\} \subseteq P$. The round 5 -set in such a 9-set can not have 3 points in $P$ since $C \cap T=\emptyset$, nor can it have 4 points in $T$, so it is a set $\{p, q, x, y, z\}$ with $p, q \in P$ and $x, y, z \in T$. Since $\{p, q, x, y, z\}$ and $\{r, s, x, y, z\}$, $r, s \in P,\{p, q\} \neq\{r, s\}$, can not both be round, there are at most 10 such round 5 -tuples. So one of these must be in $\geq 495 / 10$, so in at least 50 of our 9 -tuples. But only $\left(\begin{array}{c}10 \\ 2\end{array}\right)=45$ of these contain a given pair $p, q \in P$ and thus our assumption is false. 
So all 5-tuples in $Q$ contain a round 4-tuple. If a 5-tuple contains two round 4-tuples it is itself round, and if all 5-tuples in $Q$ are round $Q$ itself is round, so then $V$ lies on two circles and we have $R^{*}(9,5)$. Otherwise we have a 5-subset $T=\{a, b, c, d, e\} \subseteq Q$ in which $U=\{a, b, c, d\}$ is round and the other 4-tuples are not. The support of $U$ has at most 2 points in common with $P$, so in $P$ we can take a subset $P^{\prime}$ of 10 points not on the support of $U$. There are $\left(\begin{array}{c}10 \\ 4\end{array}\right)=210$ 9-tuples $\{h, i, j, k, a, b, c, d, e\}$ with $\{h, i, j, k\} \subset P^{\prime}$. Their round 5 -subsets can not contain 3 points of $P^{\prime}$ and neither 3 points of $U$. Hence they are of type $\{p, q, x, y, e\}$ with $p, q \in P^{\prime}$ and $x, y \in U$. As above a fixed triple $\{x, y, e\}$ can serve only once, so there are at most 6 such round 5-subsets; therefore one of them must be contained in $\geq 210 / 6=35$ of our 9 -sets. But since for given $p, q$ there are only $\left(\begin{array}{l}8 \\ 2\end{array}\right)=28$ 9-sets containing $p$ and $q$ this is impossible.

For the pairs $(t, s)$ treated in this section and for $(6,4)$ (see the Introduction) we thus have an upper bound for the cardinality of a set that has $R(t, s)$ but not $R^{*}(t, s)$. From Theorem 18 it will follow that there is a 12 -set with $R(7,4)$ and $R(9,5)$ not having $R^{*}(7,4)$ or $R^{*}(9,5)$. What is a sharp upper bound for these (and other) cases stays an open problem.

\section{Implied Properties in Larger Sets}

Theorem 15 Let $V$ be a set with $R(t, s)$. Let $|V| \geq w$ and $u>s$. Then $V$ has $R(w, u)$ if $\left(\begin{array}{l}w-3 \\ s-3\end{array}\right)>\left(\left(\begin{array}{l}u-3 \\ s-3\end{array}\right)-1\right)\left(\begin{array}{l}t \\ s\end{array}\right)$.

Proof. $w>t$ (by the inequality), so every $w$-subset of $V$ also has $R(t, s)$, and by Corollary 10 it contains a round $u$-set.

For example, for sufficiently large sets:

1. $R(5,4)$ entails $R(9,5), R(14,6)$ and $R(19,7)$

2. $R(6,4)$ entails $R(19,5), R(34,6)$ and $R(49,7)$

3. $R(7,4)$ entails $R(39,5), R(74,6)$ and $R(109,7)$

4. $R(8,4)$ entails $R(74,5), R(144,6)$ and $R(214,7)$.

In particular cases Theorem 11 can give a better result. It shows that in 2 . we can replace $R(19,5)$ by $R(17,5)$ and in $4 . R(74,5)$ by $R(73,5)$.

1. is no news: for sets with $N \geq 7$ points and $R(5,4)$ we have $C(1)$ (see the Introduction), so $R(6,5), R(7,6)$ and, if $N \geq 8, R(8,7)$.

2. is some news: we only knew that for sets with $\geq 78$ points $C(2)$ and thus also $R(7,5), R(8,6)$ and $R(9,7)$ follow from $R(6,4)$.

3. a set with $R(7,4)$ and at least 809 points has $R^{*}(7,4)$, which guarantees $R(9,5)$, $R(11,6)$ and $R(13,7)$. 
Theorem 16 For all $t$ and $s$ with $s>4$ and $2 s \leq t+1$ there is a number $N(t, s)$ such that every set $V$ with $R(t, s)$ and $|V| \geq N(t, s)$ either is the union of a round set and a set with $R(t-s+1, s)$ or has $C(t-s)$.

Proof. By Theorem 11 there is a number $N(t, s)$ such that all sets $V$ with $R(t, s)$ and $|V| \geq N(t, s)$ contain a round set of cardinality $n:=2\left(\begin{array}{c}t-s+1 \\ 3\end{array}\right)+s-1$. Let $V$ be such a set. Take a round $n$-set in $V$, let $C$ be its support and let $W=V \cap C$. Suppose $V$ has not $C(t-s)$. Then let $Q$ be a set of $t-s+1$ points of $V-W$. We must prove that $\mathrm{Q}$ contains a round $s$-set. Now $W$ contains a subset $X$ of cardinality $s-1$ such that none of its points is on one of the $\leq\left(\begin{array}{c}t-s+1 \\ 3\end{array}\right)$ round sets containing 3 points of $Q$.

The $t$-set $X \cup Q$ contains a round $s$-set $S$ which must contain a point of $Q$, and thus can contain at most 2 points of $X$, so it contains at least 3 points of $Q$. But then it contains no points of $X$, so $Q$ contains a round $s$-set and $V-W$ has $R(t-s+1, s)$.

\section{Less Simple Examples}

In this section we let round mean concyclic (i.e. $\mathcal{S}$ is the set of all circles). Throughout $\mathcal{C}=\left\{C_{1}, C_{2}, \ldots, C_{q}\right\}$ is a set of $q$ circles, $q \geq 3$, of which no three have a point in common but every pair intersects in 2 points. $V(\mathcal{C})$ will denote the set of their $q(q-1)$ intersection points and there are $2 q(q-1)$ pairs $\langle v, C\rangle$ with $v \in V(\mathcal{C}), C \in \mathcal{C}$ and $v \in C$. We call these pairs incidences.

We need the following observation.

Lemma 17 If a circle $D$ contains more than $q$ points of $V(\mathcal{C})$ then $D \in \mathcal{C}$.

Proof. If $D$ contains more than $q$ points of $V(\mathcal{C})$ there are at least $2 q+2$ incidences $\langle v, C\rangle$ with $v \in D$. Thus there is a $C \in \mathcal{C}$ containing more than 2 points of $D$, and $D$ must be that $C$.

Theorem $18 V(\mathcal{C})$ has property $R\left(\left\lfloor\frac{q(s-1)}{2}+1\right\rfloor, s\right)$ for all $s$ with $4 \leq s \leq 2 q-2$, but not property $R^{*}\left(\left\lfloor\frac{q(s-1)}{2}+1\right\rfloor, s\right)$.

Proof. Note that, since $s \leq 2 q-2,\left\lfloor\frac{q(s-1)}{2}+1\right\rfloor \leq q(q-1)=|V(\mathcal{C})|$. Let $W \subseteq V(\mathcal{C})$ with $|W|=\left\lfloor\frac{q(s-1)}{2}+1\right\rfloor$. The number of incidences $\langle w, C\rangle$ with $w \in W$ is $2|W| \geq q(s-1)+1$, so there is a $C \in \mathcal{C}$ containing more then $s-1$ points of $W$, which proves the first assertion.

Now suppose $V(\mathcal{C})$ has $R^{*}\left(\left\lfloor\frac{q(s-1)}{2}+1\right\rfloor, s\right)$. Then there is a $k>0$ and a set of $k$ circles that together contain all points of $V(\mathcal{C})$ with the exception of at most $\left\lfloor\frac{q(s-1)}{2}\right\rfloor-k(s-1)$. Clearly $k \leq q / 2$. Suppose $l$ of these circles belong to $\mathcal{C}$. They contain $\sum_{j=1}^{l} 2(q-j)$ points of $V(\mathcal{C})$. The remaining $k-l$ circles each contain at most $q$ points of $V(\mathcal{C})$, by the lemma, but $q \leq 2(q-j)$ if $j \leq q / 2$, so our $k$ circles contain at most $\sum_{j=1}^{k} 2(q-j)=$ $2 k(q-1)-k(k-1)$ points of $V(\mathcal{C})$.

It would follow that 


$$
q(q-1)-2 k(q-1)+k(k-1) \leq\left\lfloor\frac{q(s-1)}{2}\right\rfloor-k(s-1) .
$$

We omit the "floors" and then can rewrite this as

$$
\left(\frac{q}{2}-k\right)(2 q-s-1)+k(k-1) \leq 0 .
$$

Since $2 q-s-1 \geq 1, \frac{q}{2}-k \geq 0$ and $k>0$, it would follow that $q=2 k$ and $k=1$, contradicting $q \geq 3$.

E.g. $q=20$ gives a 380 -set with $R(31,4)$ as well as $R(371,38)$. In between: $R(81,9)$ and $R(91,10)$. With $q=51$ we find a 2550-set with, for instance, $R(1251,50)$.

Given $s$ we get the strongest property (the smallest " $t$ ") for $q=\left\lceil\frac{s+2}{2}\right\rceil$, but then also the smallest sets: 6 points with $R(5,4), 12$ points with $R(9,5)$ (and also $R(11,6)$ ), 20 points with $R(16,7)$ (and also $R(18,8)$ ), etc.

It is an easy exercise to show that the theorem with $q=3$ and $s=4$ gives the only sets with $R(5,4)$ and not $C(1)$, if we admit that one of the circles is disguised as a line (which, in fact, we could also have done in our definition of $\mathcal{C}$ ).

\section{Remarks on the 3-Dimensional Case}

Generalisation to Euclidean 3-space is not a simple matter. The reason is that, whereas two circles in the plane are the same if they have 3 points in common, two spheres in 3 -space sharing 4 (or even more) points need not be the same.

Let a set (always: of points in Euclidean 3-space) have property $S(t, s)$ if every $t$-subset contains a spherical $s$-subset $(s>4)$. Again we have the "standard" examples like: a set has $S(7,5)$ if all points with the exception of at most two are on a sphere. But there are other more or less trivial configurations: take a sphere $Q$, points $A, B, C$ not on $Q$ and 3 spheres each through $A, B$ and $C$ and intersecting $Q$ in (disjoint) circles. Every set containing $A, B$ and $C$ and moreover only points of these circles has $S(7,5)$. If this set has cardinality $\geq 13$ one of the circles contains 4 points of it, and we could eliminate this example by forbidding concyclic 4-tuples.

When we restrict ourselves to sets without concyclic 4-tuples (which includes sets in which every 4-tuple is in general position) it is not difficult to prove analogues of some of the above results. We then can use that 4 points of a spherical subset of such a set determine the support of that subset. We give some examples, with condensed or omitted proofs. The proofs of the following three theorems are almost copies of those of the Propositions 5, 6 and 7 .

Proposition 19 Let $2 s \geq t+5$. A set with $S(t, s)$ and containing no concyclic 4-set has all points except for at most $t-s$ on a sphere.

Proposition 20 Let $2 s=t+4$ and $s \geq 7$. A set with $S(t, s)$ and containing no concyclic 4-set has all points except for at most $t-s$ on a sphere.

Proposition 21 Let $2 s=t+3$ and $s \geq 8$. A set with $S(t, s)$ and containing no concyclic 4-set has all points except for at most $t-s$ on a sphere. 
Proof. Let $V$ be such a set, let $S$ be the support of a largest spherical subset of $V$ and take $W=\{1,2, \ldots, s\}$ on $V \cap S$. Suppose we have a subset $U=\{s+1, s+2, \ldots, t+1\}$ of points not on $S ;|U|=s-2 \geq 6$. First assume that $U$ is not spherical. Then $(W-\{1\}) \cup U$ contains a spherical $s$-set with 3 points in $W$ and $s-3$ points in $U$, say $\{2,3,4\} \cup U^{\prime}$. The spherical $s$-set in $(W-\{2\}) \cup U$ then contains an $(s-3)$-subset $U^{\prime \prime}$ of $U$ with $U^{\prime \prime} \neq U^{\prime}$. But then $U^{\prime} \cup U^{\prime \prime}=U$, and since $\left|U^{\prime} \cap U^{\prime \prime}\right|=s-4 \geq 4, U$ is spherical, a contradiction. If $U$ is spherical, $(W-\{1\}) \cup U$ shows that $W$ has at least two points on the support of $U$, say 2 and 3 . The same goes for $(W-\{2\}) \cup U$, so we may suppose that 1,2 and 3 are on the support of $U$. Since $U \cup\{1,2,3\}$ is spherical and has $s+1$ points, by definition of $S$ there is a point $x$ in $V \cap S, x \notin W$. Now using $W^{\prime}=\{x, 1,3,4, \ldots, s\}$ instead of $W$ we see that $W^{\prime}-\{1\} \cup U=\{x, 3,4, \ldots, s\} \cup U$ also has two points on the support of $U$. So we have 4 points on the intersection of $S$ and the support of $U$, a contradiction.

Theorem 22 Of a set with $S(t, t-1)$ and containing no concyclic 4-set all points except for at most one lie on a sphere.

Proof. The cases with $t>6$ are covered by Proposition 19, so we need only consider $S(6,5)$. Take a spherical 5 -set $S=\{1,2,3,4,5\}$. Suppose the set has two points $A, B$ not on its support. The 6 -set $\{1,2,3,4, A, B\}$ yields a spherical 5 -set $\{1,2,3, A, B\}$ (after renumbering $\{1,2,3,4\})$. Let $T$ be its support. $T$ does not contain 4 or 5 . The 6 -set $\{2,3,4,5, A, B\}$ yields a spherical 5 -set, which can not be $\{2,3,4, A, B\}$ or $\{2,3,5, A, B\}$ (since then 4 or 5 would be on $T$ ). So we can suppose it is $\{3,4,5, A, B\}$ (interchange the names of 2 and 3 if necessary). Its support $U$ does not contain 1 or 2 . Now every 5 -subset of $\{1,2,4,5, A, B\}$ has four points on one of $S, T$ or $U$, but not the fifth point. Contradiction.

As to an analogue of Theorem 9 we restrict ourselves to the case $S(7,5)$.

Theorem 23 A set of cardinality $N$ with $S(7,5)$ and containing no concyclic 4 -set contains a spherical $m$-set $(m>5)$ if $N \geq 21 m-100$.

Proof. Let $r$ be the number of spherical quintuples in the set. The number of pairs $\langle Q, S\rangle$ with $Q$ a spherical quintuple, $S$ a 7 -subset and $Q \subset S$ is $r\left(\begin{array}{c}N-5 \\ 2\end{array}\right)$, and also at least $\left(\begin{array}{c}N \\ 7\end{array}\right)$. So

$$
\left(\begin{array}{l}
N \\
7
\end{array}\right) \leq r\left(\begin{array}{c}
N-5 \\
2
\end{array}\right)
$$

The number of pairs $\langle F, Q\rangle$ with $F$ a 4 -subset, $Q$ a spherical quintuple and $F \subset Q$ is $5 r$. If every 4 -subset would be in at most $m-5$ spherical quintuples, then we would have

$$
5 r \leq(m-5)\left(\begin{array}{c}
N \\
4
\end{array}\right) \text {. }
$$

From $(*)$ and $(* *)$ however it would follow that

$$
N \leq 21 m-101 \text {. }
$$

So there is a (non-planar) 4-subset belonging to at least $m-4$ spherical quintuples. Their union is a spherical $m$-set. 


\section{References}

[1] R.H. Jeurissen and A.J.Th. Maassen, Nogmaals over Vogeltjes en Cirkels (en Designs), Wiskunde \& Onderwijs, 26 (2000), 216-226. (Dutch)

[2] E. Soetens, D. Tant and J. van Casteren, Over vogeltjes en cirkels, Wiskunde \& Onderwijs, 23 (1997), 358-365. (Dutch) 